\title{
Quasitriangularity in Hilbert Space
}

\section{CONSTANTIN APOSTOL}

\author{
Communicated by the Editors
}

Introduction. Initiating the study of quasitriangular operators in Hilbert space, P. R. Halmos [8] showed that they play an important role in the proofs of some invariant subspaces theorems. A natural question arises: How large is the class of quasitriangular operators? P. R. Halmos proved that a normal operator is quasitriangular ([8]); R. G. Douglas and C. Pearcy proved that a quasinilpotent operator is quasitriangular ([4]). The aim of this note is to show that "quasitriangularity" is a concept attached to operators with "good" spectral properties, namely to decomposable operators of C. Foiaş [6]. Thus spectral operators ([5]) and generalized scalar operators ([7]) are quasitriangular. The conjecture of R. G. Douglas and C. Pearcy [4] that an operator whose spectrum is a countable set is quasitriangular, proved first by P. R. Halmos [13], results to be true because such an operator is decomposable. B. Sz.-Nagy and C. Foias [11] proved that a contraction $T$ in a Hilbert space such that $\left\{T^{n}\right\},\left\{T^{* n}\right\}$ do not tend strongly to 0 , has a proper closed invariant subspace. It is still not known if $T$ has a proper invariant subspace if $\left\{T^{m}\right\}$ does not tend strongly to 0 . We prove that if $\left\{T^{n}\right\} \nrightarrow 0$, and $T$ has no proper invariant subspace, then $T^{*}$ is a quasitriangular operator.

P. Meyer-Nieberg [9] defined quasitriangular operators in Banach space. Since we use strongly the existence of orthogonal projections in Hilbert space, our proofs cannot be extended to Banach spaces. Anyway, it seems to be very difficult to generalize the below results to Banach spaces.

1. Preliminaries and notation. Throughout, $\mathbf{X}$ will denote a separable, complex Hilbert space with $\operatorname{dim} \mathbf{X} \geqq 2, \mathfrak{L}(\mathbf{X})$ the algebra of all bounded linear operators acting in $\mathbf{X}, \mathfrak{I}$ the set of all finite-dimensional subspaces in $\mathbf{X}$. The term subspace will mean a closed linear manifold. If $\mathbf{Y}$ is a subspace in $\mathbf{X}$, then $P_{\mathbf{Y}}$ is the orthogonal projection of $\mathbf{X}$ onto $\mathbf{Y}$.

Let $T \varepsilon \mathcal{L}(\mathbf{X})$ and $Y$ be a subspace in $\mathbf{X}$.

1.1. Definition. The subspace $\mathbf{Y}$ is called a spectral maximal space of $T$ if $T \mathbf{Y} \subset \mathbf{Y}$ and for any subspace $\mathbf{Z}(\subset \mathbf{X})$ such that $T \mathbf{Z} \subset \mathbf{Z}, \sigma(T \mid \mathbf{Z}) \subset \sigma(T \mid \mathbf{Y})$ we have $\mathrm{Z} \subset \mathrm{Y}$ ([2], Ch. I, Def. 3.1). 
1.2. Definition. The operator $T$ has the single-valued extension if the equation $(\lambda-T) f(\lambda) \equiv 0$ has the only $\mathbf{X}$-valued analytic solution $f=0$, on any domain in the complex plane ([2], Ch. I, Def. 1.1).

Suppose $T$ has the single-valued extension property and let $x \varepsilon \mathrm{X}$. Then the equation $(\lambda-T) x_{T}(\lambda)=x$, has a unique $\mathrm{X}$-valued analytic solution $x_{T}(\cdot)$ defined in a maximal open set $\rho_{T}(x)$. The complementary set of $\rho_{T}(x)$ will be denoted by $\sigma_{T}(x)$ (see [2], Ch. I, \$1).

1.3. Definition. The operator $T$ is called decomposable if for any open covering $\left\{G_{i}\right\}_{i}^{n}$ of $\sigma(T)$ there exists a system of spectral maximal spaces $\left\{Y_{i}\right\}_{i}^{n}$ of $T$ such that $\sum_{i=1}^{n} \mathbf{Y}_{i}=\mathrm{X}, \sigma\left(T \mid \mathbf{Y}_{i}\right) \subset G_{i}$ ([2], Ch. II, Def. 1.1).

For any $x \varepsilon \mathrm{X}$ denote

$$
r_{T}(x)=\inf _{\lambda} \lim _{n \rightarrow \infty}\left\|(\lambda-T)^{n} x\right\|^{1 / n}, \bar{r}_{T}(x)=\inf _{\lambda} \varlimsup_{n \rightarrow \infty}\left\|(\lambda-T)^{n} x\right\|^{1 / n}
$$

Obviously we have $\underline{r}_{T}(x) \leqq \bar{r}_{T}(x)$. If we agree that diam $\varnothing=0$, then it follows: have

1.4. Proposition. Let $T$ have the single-valued extension property. Then we

$$
\frac{1}{2} \operatorname{diam} \sigma_{T}(x) \leqq \bar{r}_{T}(x) \leqq \operatorname{diam} \sigma_{T}(x) .
$$

Proof. Let $x \varepsilon \mathrm{X}, x \neq 0$. By 2, Ch. I, Prop. 1.2, $\sigma_{T}(x) \neq \varnothing$. Choose $\lambda_{0} \varepsilon$ $\sigma_{T}(x)$ such that $\sigma_{T}(x) \subset\left\{\mu:\left|\mu-\lambda_{0}\right| \leqq \operatorname{diam} \sigma_{T}(x)\right\}=\sigma$. Since $x_{T}(\mu)=R(\mu ; T) x$, if $|\mu|>\| T||$ one obtains

$$
x_{T}(\mu)=\sum_{n=0}^{\infty} \frac{\left(T-\lambda_{0}\right)^{n} x}{\left(\mu-\lambda_{0}\right)^{n+1}}
$$

for large $|\mu|$. Because $x_{T}(\mu), \mu \notin \sigma$ can be obtained by direct extension from the right hand of the above relation we obtain

$$
\varlimsup_{n \rightarrow \infty} \frac{\|\left(T-\lambda_{0}\right)^{n} x||^{1 / n}}{\left|\mu-\lambda_{0}\right|}<1, \quad \text { if } \quad\left|\mu-\lambda_{0}\right|>\operatorname{diam} \sigma_{T}(x)
$$

which implies $\varlimsup_{n \rightarrow \infty}\left\|\left(\lambda_{0}-T\right)^{n} x\right\|^{1 / n} \leqq \operatorname{diam} \sigma_{T}(x)$ and henceforth $\bar{r}_{T}(x) \leqq$ $\operatorname{diam} \sigma_{T}(x)$. Conversely, if $\lambda_{0}$ is chosen such that $\overline{\lim }_{n \rightarrow \infty}\left\|\left(\lambda_{0}-T\right)^{n} x\right\|^{1 / n}<$ $\bar{r}_{T}(x)+\epsilon$, then the function

$$
f(\mu)=\sum_{n=0}^{\infty} \frac{\left(T-\lambda_{0}\right)^{n} x}{\left(\mu-\lambda_{0}\right)^{n+1}}
$$

is well-defined and analytic in $\left\{\mu:\left|\mu-\lambda_{0}\right|>\vec{r}_{T}(x)+\epsilon\right\}$. An easy calculation shows that we have $(\mu-T) f(\mu)=x$; thus $\rho_{T}(x) \subset\left\{\mu:\left|\mu-\lambda_{0}\right|>\bar{r}_{T}(x)+\epsilon\right\}$. It results $\sigma_{T}(x) \subset\left\{\mu:\left|\mu-\lambda_{0}\right| \leqq \bar{r}_{T}(x)\right\}$ and hence diam $\sigma_{T}(x) \leqq 2 \bar{r}_{T}(x)$. To conclude the proof we have to remark that if $x=0$, then $\bar{r}_{T}(x)=0, \sigma_{T}(x)=\varnothing$.

1.5. Lemma. Let $\mathrm{X}_{n} \varepsilon$ g such that $P_{\mathrm{X}_{n}} \stackrel{\rightarrow}{\rightarrow} I$ (= the identity operator) and let $\mathrm{Y}_{\varepsilon} \mathcal{J}$. Then there exists $\mathrm{Y}_{n} \varepsilon \mathcal{J}, \mathrm{Y}_{n} \supset \mathrm{Y}$ such that $\left\|P_{\mathbf{X}_{n}}-P_{\mathbf{Y}_{n}}\right\| \rightarrow 0$. 
Proof. Let us set $\mathbf{Z}_{n}=\mathbf{X}_{n} \ominus P_{\mathbf{X}_{n}} \mathbf{Y}, \mathbf{Y}_{n}=Z_{n}+\mathbf{Y}, \epsilon_{n}=\left\|\left(I-P_{\mathbf{X}_{n}}\right) \mid \mathbf{Y}\right\|$. We have $\epsilon_{n} \rightarrow 0$ and for any $z_{n} \varepsilon Z_{n}, y \varepsilon \mathrm{Y}$

$$
\left(z_{n}, y\right)=\left(P_{\mathbf{x}_{n}} z_{n}, y\right)=\left(z_{n}, P \mathbf{x}_{n} y\right)=0 .
$$

This shows that $Z_{n}$ and $\mathbf{Y}$ are orthogonal subspaces. Now define the operator $S_{n}: \mathrm{X} \rightarrow \mathrm{X}_{n}$ by the equation

$$
S_{n} x=\left\{\begin{array}{l}
0, x \varepsilon Y_{n}^{1}, \\
z_{n}+P_{\mathbf{X}_{n}} y, x=z_{n}+y \varepsilon Y_{n} .
\end{array}\right.
$$

We have

$$
\left\|S_{n}-P_{\mathbf{X}_{n}}\right\|=\sup _{\substack{\left\|z_{n}+\nu\right\|=1 \\ 2 n \in Z_{n}, y \in \mathbf{X}}}\left\|y-P_{\mathbf{X}_{n}} y\right\| \leqq \sup _{\substack{\|\| \leq 1 \\ y \in \mathbf{X}}}\left\|\left(I-P_{\mathbf{X}_{n}}\right) y\right\|=\epsilon_{n} ;
$$

thus $\lim _{n \rightarrow \infty}\left\|S_{n}-P_{\mathbf{Y}_{n}}\right\|=0$. Let $S_{n}=U_{n}\left|S_{n}\right|$ be the polar representation of $S_{n}$. We have

$$
\left|S_{n}\right|^{2}-P_{\mathbf{Y}_{n}}=\left(S_{n}^{*}-P_{\mathbf{Y}_{n}}\right)\left(S_{n}-P_{\mathbf{Y}_{n}}\right)+P_{\mathbf{Y}_{n}} S_{n}+S_{n}^{*} P_{\mathbf{Y}_{n}}-2 P_{\mathbf{Y}_{n}}
$$

which implies successively

$$
\begin{aligned}
\left\|\left|S_{n}\right|^{2}-P_{\mathbf{Y}_{n}}\right\| \rightarrow 0 & \Rightarrow\left\|\left|S_{n}\right|-P_{\mathbf{Y}_{n}}\right\| \\
\Rightarrow\left\|U_{n}-P_{\mathbf{Y}_{n}}\right\| & \leqq\left\|U_{n}\left(P_{\mathbf{Y}_{n}}-\left|S_{n}\right|\right)\right\| ! \\
& +\left\|S_{n}-P_{\mathbf{Y}_{n}}\right\| \rightarrow 0 .
\end{aligned}
$$

Using the relations $\left\|U_{n}-P_{\mathbf{Y}_{n}}\right\| \rightarrow 0, U_{n} \mathbf{Y}_{n}=\mathbf{X}_{n}$ we obtain $P_{\mathbf{X}_{n}}=U_{n} P_{\mathbf{Y}_{n}} U_{n}^{*}$ and hence

$$
\lim _{n \rightarrow \infty}\left\|P_{\mathbf{X}_{n}}-P_{\mathbf{Y}_{n}}\right\|=\lim _{n \rightarrow \infty}\left\|U_{n} P_{\mathbf{Y}_{n}} U_{n}-P_{\mathbf{Y}_{n}}\right\|=0 .
$$

2. The modulus of quasitriangularity. Let $T \in \mathcal{E}(\mathbf{X})$. The modulus of quasitriangularity $q(T)$ of $T$ will be by definition the number

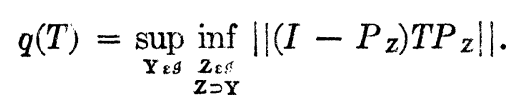

2.1. Proposition. The map $T \rightarrow q(T)$ is continuous; for any $T \varepsilon \&(\mathbf{X})$ there exists a sequence of increasing subspaces $\left\{\mathbf{X}_{n}\right\}_{1}^{\infty} \subset$ g such that $P_{\mathbf{X}_{n}} \dot{\rightarrow} I$, $\left\|\left(I-P_{\mathbf{X}_{n}}\right) T P_{\mathbf{x}_{n}}\right\| \rightarrow q(T)$. We have the equality

$$
q(T)=\inf \left\{\varlimsup_{n \rightarrow \infty}\left\|\left(I-P_{\mathbf{Y}_{n}}\right) T P_{\mathbf{Y}_{n}}\right\|:\left\{\mathbf{Y}_{n}\right\}^{\infty} \stackrel{\subset}{ } \subset \mathcal{g}, P_{\mathbf{Y}_{n}} \dot{\rightarrow} I\right\} .
$$

Proof. Let $T, S \varepsilon \mathfrak{L}(\mathbf{X})$. For any $\mathbf{Y}, \mathbf{Z} \varepsilon$ J, $\mathbf{Z} \supset \mathbf{Y}$ we have

$$
\left\|\left(I-P_{\mathbf{z}}\right) T P_{\mathbf{z}}\right\| \leqq\left\|\left(I-P_{\mathbf{z}}\right) S P_{\mathbf{z}}\right\|+\|T-S\|
$$


which implies

$$
\inf _{\substack{Z_{\mathbf{z}} \\ \mathbf{Z} \supset \mathbf{Y}}}\left\|\left(I-P_{\mathrm{z}}\right) T P_{\mathbf{z}}\right\| \leqq \inf _{\substack{\mathbf{Z}_{\mathbf{z}} \\ \mathbf{Z} \supset \mathbf{Y}}}\left\|\left(I-P_{\mathrm{z}}\right) S P_{\mathbf{z}}\right\|+\|T-S\|
$$

and $q(T) \leqq q(S)+\|T-S\|$. It follows the inequality

$$
|q(T)-q(S)| \leqq\|T-S\|
$$

whence the continuity of $T \rightarrow q(T)$ results. Now by the definition of $q(T)$ we can choose $Y_{n} \&$ I such that

Since

$$
\lim _{n \rightarrow \infty} \inf _{\substack{\mathbf{Z}_{\mathbf{z}} \\ \mathbf{z} \supset \mathbf{Y}_{n}}}\left\|\left(I-P_{\mathbf{z}}\right) T P_{\mathbf{z}}\right\|=q(T) .
$$

$$
\inf _{\substack{\mathbf{Z}_{\mathbf{z}} \\ \mathbf{z} \supset \mathbf{X}}}\left\|\left(I-P_{\mathbf{z}}\right) T P_{\mathbf{z}}\right\|
$$

increases with $\mathrm{Y}$ we may suppose we have $\mathrm{Y}_{n} \subset \mathrm{Y}_{n+1}, P_{\mathrm{Y}_{n}} \dot{\rightarrow} I$. By induction we can determine $X_{n} \varepsilon$ I such that

$\mathbf{X}_{n+1} \supset \mathbf{Y}_{n+1}+\mathbf{X}_{n}, \mathbf{X}_{1}=\mathbf{Y}_{1}$ and

We have

$$
\left\|\left(I-P_{\mathbf{X}_{n+1}}\right) T P_{\mathbf{X}_{n+1}}\right\|<\inf _{\mathbf{z}_{n+1}+\mathbf{X}_{n}}\left\|\left(I-P_{z}\right) T P_{z}\right\|+\frac{1}{n}
$$

$$
\begin{aligned}
\inf _{\substack{\mathbf{z} g \\
\mathbf{z} \supset \mathbf{Y}_{n}}}\left\|\left(I-P_{\mathbf{z}}\right) T P_{z}\right\| & \left.\leqq \| I-P_{\mathbf{X}_{n+1}}\right) T P_{\mathbf{X}_{n+1}} \| \\
& <\inf _{\substack{\mathbf{Z}_{z g} \\
\mathbf{Z} \supset \mathbf{Y}_{n+1} \vee \mathbf{X}_{n}}}\left\|\left(I-P_{\mathbf{z}}\right) T P_{\mathbf{z}}\right\|+\frac{1}{n} \leqq q(T)+\frac{1}{n}
\end{aligned}
$$

and this implies

$$
\lim _{n \rightarrow \infty}\left\|\left(I-P_{\mathbf{X}_{n}}\right) T P_{\mathbf{X}_{n}}\right\|=q(T) .
$$

The existence of $\left\{\mathbf{X}_{n}\right\}_{\circ}^{\infty} \subset \mathcal{J}$ with the above properties implies

$$
q(T) \geqq \inf \left\{\overline{\lim }_{n \rightarrow \infty}\left\|\left(I-P_{\mathbf{Y}_{n}}\right) T P_{\mathbf{Y}_{n}}\right\|:\left\{\mathbf{Y}_{n}\right\}_{1}^{\infty} \subset \mathcal{g}, P_{\mathbf{Y}_{n}} \stackrel{s}{\rightarrow} I\right\} .
$$

Now let $\left\{\mathrm{Y}_{n}\right\}_{1}^{\infty} \subset \mathcal{g}, \mathrm{Y}_{\varepsilon} \mathcal{g}, P_{\mathrm{Y}_{n}} \stackrel{\leftrightarrow}{\rightarrow} I$. By Lemma 1.5 we can find $Z_{n} \varepsilon \mathcal{g}, Z_{n} \supset \mathrm{Y}$ such that $\left\|P_{\mathbf{Y}_{n}}-P_{Z_{n}}\right\| \rightarrow 0$. Then

$$
\inf _{\substack{Z_{\varepsilon S} \\ Z \supset \mathbf{Y}}}\left\|\left(I-P_{Z}\right) T P_{Z}\right\| \leqq \varlimsup_{n \rightarrow \infty}\left\|\left(I-P_{Z_{n}}\right) T P_{Z_{n}}\right\|=\varlimsup_{n \rightarrow \infty}\left\|\left(I-P_{\mathbf{Y}_{n}}\right) T P_{\mathbf{Y}_{n}}\right\| .
$$

Consequently

$$
q(T)=\sup _{\mathbf{Y} \varepsilon \mathcal{S}} \inf _{\substack{\mathbf{Z}_{\mathfrak{g}} \\ \mathbf{Z} \supset \mathbf{Y}}}\left\|\left(I-P_{\mathbf{Z}}\right) T P_{\mathbf{Z}}\right\| \leqq \varlimsup_{n \rightarrow \infty}\left\|\left(I-P_{\mathbf{Y}_{n}}\right) T P_{\mathbf{Y}_{n}}\right\| .
$$

Since $\left\{\mathrm{Y}_{n}\right\}^{\infty} \subset \mathcal{} \mathcal{}$ J,$P_{\mathbf{Y}_{n}} \rightarrow I$ is arbitrary, the proof is finished. 
2.2. Theorem. Let $T \varepsilon \&(\mathbf{X})$ and $\mathrm{Y}$ be an invariant subspace of $T$. Then we have

$$
q(T) \leqq \max \left\{q(T \mid \mathrm{Y}), \quad q\left(\left(I-P_{\mathbf{Y}}\right) T \mid \mathrm{Y}^{\perp}\right)\right\} .
$$

Proof. Denote $P_{\mathbf{Y}}=P, P_{\mathrm{Y} \perp}=Q$ and choose $\mathbf{X}_{n}, \mathbf{X}_{n} \varepsilon \mathcal{J}, \mathbf{X}_{n} \subset \mathrm{Y}, \mathbf{Y}_{n} \subset \mathbf{Y}^{\perp}$ such that

$P_{\mathbf{X}_{n}} \stackrel{\leftrightarrow}{\rightarrow} P, P_{\mathbf{Y}_{n}} \stackrel{\leftrightarrow}{\rightarrow} Q,\left\|\left(P-P_{\mathbf{X}_{n}}\right) T P_{\mathbf{X}_{n}}\right\| \rightarrow q(T \mid \mathrm{Y})$,

$$
\left\|\left(Q-P_{\mathbf{Y}_{n}}\right) T P_{\mathbf{Y}_{n}}\right\| \rightarrow q\left(Q T \mid \mathbf{Y}^{\perp}\right) .
$$

For any $x \& \mathbf{X}$ we have

$$
\begin{aligned}
& \left\|\left(I-P_{\mathbf{X}_{m}+\mathbf{Y}_{n}}\right) T P_{\mathbf{X}_{m}+\mathbf{Y}_{n}} x\right\|^{2} \\
& \quad=\left\|\left(P-P_{\mathbf{X}_{m}}\right) T\left(P_{\mathbf{X}_{m}}+P_{\mathbf{Y}_{n}}\right) x\right\|^{2}+\left\|\left(Q-P_{\mathbf{Y}_{n}}\right) T\left(P_{\mathbf{X}_{m}}+P_{\mathbf{Y}_{m}}\right) x\right\|^{2} .
\end{aligned}
$$

Using the relation $\left(Q-P_{\mathbf{Y}_{n}}\right) T P_{\mathbf{X}_{m}}=0$ and choosing a sequence $m_{n} \nearrow$ is such that $\left\|\left(P-P_{\mathbf{R}_{n}}\right) T P_{\mathbf{Y}_{n}}\right\|<1 / n$ where $\mathbf{R}_{n}=\mathbf{X}_{m_{n}}$, we obtain

$$
\begin{aligned}
& \left\|\left(I-P_{\mathbf{R}_{n}+\mathbf{Y}_{n}}\right) T P_{\mathbf{R}_{n}+\mathbf{Y}_{n}} x\right\|^{2} \\
& \leqq\left(\left\|\left(P-P_{\mathbf{R}_{n}}\right) T P_{\mathbf{R}_{n}} x\right\|-\frac{\|x\|}{n}\right)^{2}+\left\|\left(Q-P_{\mathbf{Y}_{n}}\right) T P_{\mathbf{Y}_{n}} x\right\|^{2} \\
& \left.\leqq\left(\| P-P_{\mathbf{R}_{n}}\right) T P_{\mathbf{R}_{n}}\|\| P_{\mathbf{R}_{n}} x \|^{2}+\frac{\|x\|}{n}\right)^{2}+\left\|\left(Q-P_{\mathbf{Y}_{n}}\right) T P_{\mathbf{Y}_{n}}\right\|\left\|P_{\mathbf{Y}_{n}} x\right\|^{2} \\
& \leqq\left(\max \left\{\left\|\left(P-P_{\mathbf{R}_{n}}\right) T P_{\mathbf{R}_{n}}\right\|,\left\|\left(Q-P_{\mathbf{Y}_{n}}\right) T P_{\mathbf{Y}_{n}}\right\|\right\}\right)^{2}\|x\|^{2} \\
& +\left(2\left\|\left(P-P_{\mathbf{R}_{n}}\right) T P_{\mathbf{R}_{n}}\right\|+\frac{1}{n}\right) \frac{\|x\|^{2}}{n} \text {. }
\end{aligned}
$$

Since $P_{\mathbf{R}_{n}+\mathbf{Y}_{n}} \rightarrow I$,

$$
q(T) \leqq \varlimsup_{n \rightarrow \infty}\left\|\left(I-P_{\mathbf{R}_{n}+\mathbf{Y}_{n}}\right) T P_{\mathbf{R}_{n}+\mathbf{Y}_{n}}\right\| \leqq \max \left\{q(T \mid \mathbf{Y}), q\left(Q T \mid \mathbf{Y}^{\perp}\right)\right\}
$$

2.2. Corollary. Let $T \varepsilon \mathcal{L}(\mathbf{X})$ and $\left\{X_{n}\right\}_{1}^{\infty}$ be an increasing sequence of invariant subspaces of $T$ such that $\vee_{n=1}^{\infty} \mathbf{X}_{n}=\mathbf{X}$. Then the inequality

$$
q(T) \leqq \sup _{1 \leqq n<\infty} q\left(P_{\mathbf{X}_{n} \ominus \mathbf{X}_{n-1}} T \mid \mathbf{X}_{n} \ominus \mathbf{X}_{n-1}\right)
$$

holds (with $\mathrm{X}_{0}=\{0\}$ ).

Proof. It is an easy matter to find $\mathrm{Y}_{n} \varepsilon \mathcal{g}, \mathrm{Y}_{n} \subset \mathrm{X}_{n}$,

$$
\mathbf{Y}_{n+1} \supset \mathbf{Y}_{n}, P_{\mathbf{Y}_{n}} \stackrel{\leftrightarrow}{\rightarrow} I,\left\|\left(P_{\mathbf{X}_{n}}-P_{\mathbf{Y}_{n}}\right) T P_{\mathbf{Y}_{n}}\right\|<q\left(T \mid \mathbf{Y}_{n}\right)+\frac{1}{n} .
$$

Then

$$
q(T) \leqq \varlimsup_{n \rightarrow \infty}\left\|\left(I-P_{\mathbf{Y}_{n}}\right) T P_{\mathbf{Y}_{\mathbf{n}}}\right\|=\varlimsup_{n \rightarrow \infty}\left\|\left(P_{\mathbf{X}_{n}}-P_{\mathbf{Y}_{n}}\right) T P_{\mathbf{Y}_{n}}\right\| \leqq \varlimsup_{n \rightarrow \infty} q\left(T \mid \mathbf{X}_{n}\right)
$$


Applying Theorem 2.1 and a finite induction we have also

$$
\begin{aligned}
q\left(T \mid \mathbf{X}_{n}\right) & \leqq \max _{1 \leq i \leq n} q\left(P_{\mathbf{X}_{i} \ominus \mathbf{X}_{i-1}} \mid \mathbf{X}_{i} \ominus \mathbf{X}_{i-1}\right) \\
& \leqq \sup q\left(P_{\mathbf{X}_{i} \ominus \mathbf{X}_{i-1}} T \mid \mathbf{X}_{i} \ominus \mathbf{X}_{i-1}\right)
\end{aligned}
$$

and this implies our inequality.

2.3. Lemma. Let $x$ be a cyclic vector of the operator $T \& \&(X)$. Then we have $q(T) \leqq \tau_{r}(x)$.

Proof. Let $\epsilon>0$. Since $q(T)=q(\lambda-T)$ and $x$ is a cyclic vector for $\lambda-T$, we may suppose that we have

$$
\lim _{n \rightarrow \infty}\left\|T^{n} x\right\|^{1 / n}<\tau_{T}(x)+\epsilon .
$$

If $\left\{e_{1}, e_{2}, \cdots\right\}$ is the orthonormalization of $\{x, T x, \cdots\}$ and $\mathbf{X}_{k}=$ c.l.m. $\left\{e_{1}, \cdots, e_{k}\right\}$ we have $\left(\mathbf{X}_{0}=\{0\}\right)$

$$
\begin{gathered}
\left\|\left(I-P_{\mathbf{x}_{k}}\right) T P_{\mathbf{x}_{k}}\right\|=\left\|\left(P_{\mathbf{x}_{k+1}}-P_{\mathbf{x}_{k}}\right) T\left(P_{\mathbf{x}_{k}}-P_{\mathbf{x}_{k-1}}\right)\right\|=\left|\left(T e_{k}, e_{k+1}\right)\right|, \\
\left(T^{k} e_{1}, e_{k+1}\right)=\left(T e_{1}, e_{2}\right) \cdots\left(T e_{k}, e_{k+1}\right) .
\end{gathered}
$$

Since $e_{1}=\alpha x$ for some complex $\alpha$ and we have

$$
\lim _{n \rightarrow \infty}\left|\left(T^{n} e_{1}, e_{n+1}\right)\right|^{1 / n} \leqq \underset{\lim _{n \rightarrow \infty}}{ }\left\|T^{n} x\right\|^{1 / n}<r_{T}(x)+\epsilon
$$

we can find an increasing sequence $\{k(n)\}$ of natural numbers such that

$$
\left|\left(T e_{k(n)}, e_{k(n)+1}\right)\right|<r_{T}(x)+\epsilon+\frac{1}{n}
$$

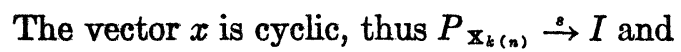

$$
q(T) \leqq \varlimsup_{n \rightarrow \infty}||\left(I-P_{\mathbf{X}_{k(n)}}\right) T P_{\mathbf{X}_{k(n)}} \|=\varlimsup_{n \rightarrow \infty}\left|\left(T e_{k(n)}, e_{k(n)+1}\right)\right| \leqq r_{T}+\epsilon .
$$

Because $\epsilon>0$ is arbitrary we obtain $q(T) \leqq r_{T}(x)$.

2.4. Theorem. Let $T \varepsilon \&(\mathrm{X})$ and $\mathrm{Y}$ be an invariant subspace of $T$. Then for any $x \varepsilon \mathrm{X}$ we have

$$
q\left(T \mid\left(\mathrm{Y} \vee\left\{T^{n} x\right\}_{0}^{\infty}\right)\right) \leqq \max \left\{q(T \mid \mathrm{Y}), r_{T}(x)\right\}
$$

Proof. Remark first that $\mathrm{Y} \vee\left\{T^{n} x\right\}_{0}^{\infty}$ is an invariant subspace of $T$. Without loss of generality we may suppose $\mathrm{Y} \vee\left\{T^{n} x\right\}_{0}^{\infty}=\mathrm{X}$. By Theorem 2.2 we have $q(T) \leqq \max \left\{q(T \mid \mathrm{Y}), q\left(\left(I-P_{\mathbf{Y}}\right) T \mid \mathrm{Y}^{\perp}\right)\right\}$. If we denote $S=\left(I-P_{\mathbf{Y}}\right) T \mid \mathrm{Y}^{\perp}$, then it is easy to see that $\left(I-P_{\mathrm{Y}}\right) x$ is a cyclic vector for $S$. Then

$$
\begin{aligned}
q(S) \leqq r_{S}\left(\left(I-P_{\mathbf{Y}}\right) x\right) & =\inf _{\lambda} \lim _{n \rightarrow \infty}\left\|\left(I-P_{\mathbf{Y}}\right)(\lambda-T)^{n}\left(I-P_{\mathbf{Y}}\right) x\right\|^{1 / n} \\
& \leqq \inf _{\lambda} \lim _{n \rightarrow \infty}\left\|\left(I-P_{\mathbf{Y}}\right)(\lambda-T)^{n} x\right\|^{1 / n} \leqq r_{T}(x) .
\end{aligned}
$$


Denote now by $\mathcal{J}(T, \epsilon)$ the set of all invariant subspaces $\mathrm{Y}$ of $T$ such that $q(T \mid \mathrm{Y}) \leqq \epsilon(\epsilon \geqq 0)$.

2.5. Proposition. The set $g(T, \epsilon)$ is inductively ordered by the inclusion. If $\mathbf{Y}$ is a maximal element in $\mathrm{g}(T, \epsilon)$, then any $x \in \mathbf{X}$ such that $\tau_{T}(x) \leqq \epsilon$ is contained in $\mathbf{Y}$.

Proof. Let $\left\{Y_{i}\right\}_{i \varepsilon I}$ be a totally ordered subset in $\mathcal{J}(t, \epsilon)$. Since $\mathbf{X}$ is separable we may reduce to the case $I=\{1,2, \cdots\}, \mathbf{Y}_{n} \subset \mathbf{Y}_{n+1}$. Denoting $\mathbf{Y}=V_{n=1}^{\infty} \mathbf{Y}_{n}$ we have to show the inequality $q(T \mid Y) \leqq \epsilon$. Let $\left\{e_{1}, e_{2}, \cdots\right\}$ be an orthonormal basis in $Y$ and choose $\mathbf{X}_{n} \varepsilon$ g, $\mathbf{X}_{n} \subset Y_{n},\left\|P_{X_{n}} e_{j}-P_{Y_{n}} e_{j}\right\|<1 / n, j=1, \cdots, n$, $\left\|\left(P_{\mathbf{Y}_{\mathrm{n}}}-P_{\mathrm{X}_{\mathrm{n}}}\right) T P_{\mathrm{X}_{\mathrm{n}}}\right\|<\epsilon+1 / n$. We have $P_{\mathrm{X}_{\mathrm{n}}} \stackrel{\dot{\rightarrow}}{\rightarrow} P_{\mathrm{Y}}$,

$$
q(T \mid \mathbf{Y}) \leqq \varlimsup_{n \rightarrow \infty}\left\|\left(P_{\mathbf{Y}}-P_{\mathbf{X}_{n}}\right) T P_{\mathbf{X}_{n}}\right\|=\varlimsup_{n \rightarrow \infty}\left\|\left(P_{\mathbf{Y}_{n}}-P_{\mathbf{X}_{n}}\right) T P_{\mathbf{X}_{n}}\right\| \leqq \epsilon .
$$

If $\mathrm{Y}$ belongs to $g(T, \epsilon)$ and $r_{T}(x) \leqq \epsilon$ we have $Y \vee\left\{T^{n} x\right\}_{0}^{\infty} \supset \mathrm{Y}$ and by Theorem 2.4. $q\left(T \mid\left(\mathrm{Y} \vee\left\{T^{m} x\right\}_{0}^{\infty}\right)\right) \leqq \epsilon$. If $\mathrm{Y}$ is maximal, then $\mathrm{Y} \vee\left\{T^{m} x\right\}_{0}^{\infty}=\mathrm{Y}$ and $x \varepsilon \mathrm{Y}$.

3. Quasitriangularity of decomposable operators. An operator $T \in \mathcal{L}(\mathbf{X})$ is called quasitriangular if its modulus of quasitriangularity is $\mathbf{0}$. Halmos [8] proved that the conditions (1) $q(T)=0$, (2) there exists $\mathbf{X}_{n} \varepsilon g$, $P_{\mathbf{x}_{n}} \nearrow I,\left\|\left(I-P_{\mathbf{x}_{n}}\right) T P_{\mathbf{x}_{n}}\right\| \rightarrow 0$, (3) there exists $\mathbf{x}_{n} \varepsilon$ g, $P_{\mathbf{x}_{n}} \dot{\rightarrow} I$, $\left\|\left(I-P_{\mathbf{x}_{n}}\right) T P_{\mathbf{X}_{n}}\right\| \rightarrow 0$ are pairwise equivalent. The equivalence can be derived also from our Proposition 2.1.

3.1. Theorem. Any decomposable operator $T_{\varepsilon} \mathfrak{L}(\mathbf{X})$ is quasitriangular.

Proof. Let $\epsilon>0$ and take a maximal element $Y$ in $\mathcal{J}(T, \epsilon)$ (via Proposition 2.5 and Zorn's Lemma). Since $T$ is decomposable it has the single-valued extension property ([2], Ch. 2, Cor. 1.4). Let $\left\{G_{i}\right\}$ be an open covering of $\sigma(T)$ such that $\operatorname{diam} G_{i} \leqq \epsilon, i=1, \cdots, n$. If $\left\{Y_{i}\right\}_{1}^{n}$ is a system of spectral maximal spaces of $T$ such that $\sigma\left(T \mid \mathrm{Y}_{i}\right) \subset G_{i}, \sum_{i=1}^{n} \mathbf{Y}_{i}=\mathrm{X}$, then for any $x \varepsilon \mathrm{Y}_{i}$ we have (see Proposition 1.4)

$$
\tau_{T}(x) \leqq \bar{r}_{T}(x) \leqq \operatorname{diam} \sigma_{T}(x) \leqq \operatorname{diam} \sigma\left(T \mid \mathbf{Y}_{i}\right) \leqq \epsilon
$$

and by Proposition 2.5 we infer $x \varepsilon \mathrm{Y}$. It follows $\mathrm{Y} \supset \sum_{i-1}^{n} \mathbf{Y}_{i}=\mathbf{X}$ and hence $\mathbf{Y}=\mathbf{X}, q(T)=q(T \mid \mathbf{Y}) \leqq \epsilon, \forall \epsilon>0$. This implies $q(T)=0$ and $T$ is quasitriangular.

3.2. Corollary. Let $T \in \mathcal{L}(\mathbf{X})$. If $T$ is spectral [5], or generalized scalar, or $\sigma(T)$ is completely disconnected (in particular if $\sigma(T)$ is a countable set), then it is a quasitriangular operator.

Proof. In all the above cases $T$ is decomposable ([2], Ch. II, Example 1.6, Ch. III Example 1.20 and Th. 1.9) and we can apply Theorem 3.1.

3.3. Proposition. Let $T \in \mathcal{L}(\mathrm{X})$ such that for any $\epsilon>0$ the equality

$$
\mathbf{X}=\operatorname{c.l} . \mathrm{m} .\left\{x \varepsilon \mathrm{X}: r_{T}(x) \leqq \epsilon\right\}
$$

holds. Then $T$ is a quasitriangular operator. 
Proof. Let $\mathrm{Y}$ be a maximal element in $g(T, \varepsilon)$ and $x \& \mathrm{X}, r_{T}(x) \leqq є \mathrm{By}$ Proposition 2.5 we have $x$ \& $\mathrm{X}$; thus $\mathrm{X}=\mathrm{X}, q(T)=q(T \mid \mathrm{Y}) \leqq \epsilon$. Since $\epsilon>0$ is arbitrarily chosen we deduce $q(T)=0$.

3.4. Corollary. Let $T \& \mathcal{L}(\mathbf{X}), S \varepsilon \mathfrak{L}\left(\mathbf{X}_{1}\right), V \in \mathcal{L}\left(\mathbf{X}_{1}, \mathbf{X}\right)$ (with $\mathbf{X}_{1}$ a Hitbert space) such that $\overline{V X}_{1}=\mathrm{X}, T V=V S$ and $S$ is decomposable. Then $T$ is a quasitriangular operator.

Proof. Let us set

$$
\mathbf{X}_{\mathbf{c}}^{1}=\left\{x \varepsilon \mathbf{X}_{1}: \bar{r}_{T}(x) \leqq \epsilon\right\} \text {. }
$$

Since $S$ is decomposable it has the single-valued extension property. Using Proposition 1.4. we obtain

$$
\mathbf{X}_{\epsilon}^{1}=\left\{x \varepsilon \mathbf{X}_{1}: \operatorname{diam} \sigma_{s}(x) \leqq 2 \epsilon\right\}
$$

For any $\epsilon>0$ we have c.l.m. $\mathbf{X}_{\varepsilon}^{1}=\mathbf{X}$ (see Definition 1.3). Let $x \varepsilon \mathbf{X}_{\epsilon}^{1}$ and choose a complex $\lambda_{0}$ such that

$$
\bar{r}_{S}(x)=\varlimsup_{n \rightarrow \infty}\left\|\left(\lambda_{0}-S\right)^{n} x\right\|^{1 / n}
$$

We have

$$
\underline{\lim }_{n \rightarrow \infty}\left\|\left(\lambda_{0}-T\right)^{n} V x\right\|^{1 / n}=\underline{\lim _{n \rightarrow \infty}}\left\|V\left(\lambda_{0}-S\right)^{n} x\right\|^{1 / n} \leqq \vec{r}_{S}(x) \leqq \epsilon
$$

which implies $r_{T}(V x) \leqq \epsilon$. It follows

$$
\text { c.l.m. }\left\{x \varepsilon \mathrm{X}: r_{T}(x) \leqq \epsilon\right\} \supset \text { c.l.m. } V \mathbf{X}_{\varepsilon}^{1}=\mathbf{X}
$$

and applying Proposition 3.3, $T$ results to be quasitriangular.

3.5. Theorem. Let $T \varepsilon \mathcal{L}(\mathbf{X}),\|T\| \leqq 1$ be such that $T^{m} \stackrel{\rightarrow}{\rightarrow} 0$. If $T$ has no proper invariant subspace, then $T^{*}$ is a quasitriangular operator.

Proof. Let $A$ be defined by the equation

$$
(A x, x)=\lim _{n \rightarrow \infty}\left(T^{* n} T^{n} x, x\right) \text {. }
$$

We have $\mid A^{1 / 2} T x\|=\| A^{1 / 2} x \|, \forall x \varepsilon \mathrm{X}, A=A^{*} \neq 0$. Since $\operatorname{Ker} A$ and $\overline{T \mathrm{X}}$ are invariant subspaces of $T, \operatorname{Ker} A=\{0\}, \overline{T \mathbf{X}}=\mathbf{X}$. Let $\mathbf{X}_{1}$ be the completion of $\mathbf{X}$ in the norm $\|\cdot\|_{1}$ derived from the scalar product $x, y \rightarrow(A x, y)$. Using the equalities $\|T x\|_{1}=\|x\|_{1}, \forall x \varepsilon \mathbf{X}, \overline{T X}=\mathbf{X}$ and the continuity of the inject:on $V: \mathbf{X} \rightarrow \mathbf{X}_{1}(V x=x)$ we can extend $T$ by continuity to a unitary operator $S \varepsilon \&\left(\mathbf{X}_{1}\right)$ such that $S V=V T$. But this implies $T^{*} V^{*}=V^{*} S^{*}, \vec{V}^{*} \mathbf{X}_{1}=\mathbf{X}$ (via Ker $V=\{0\}$ ) and $S^{*}$ being decomposable (cf. [2], Ch. II, Example 1.6) we can apply Corollary 3.4 .

Remark. In Theorem 3.5 the assumption $\|T\| \leqq 1$ can be replaced by $\sup _{1 \leq n<\infty}\left\|T^{n}\right\|<\infty$. The operator $A$ in our proof will be defined in this case using Banach limits (see [12], Ch. II, Prop. 5.3). 


\section{REFERENCES}

1. C. Apostol, Hypercommutativity and invariant subspaces, Rev. Roum. Math. Pures et Appl.

2. I. Colojoară \& C. Foiaş, Theory of generalized spectral operators, Gordon and Breach Sci. Publ. New York, 1968.

3. R. G. Douglas \& C. Pearcy, A characterization of thin operators, Acta Sci. Math. Szeged 29 (1968), 295-298.

4. R. G. Douglas \& C. Pearcy, A note on quasitriangular operators, Duke Math. J. 37 (1970), 177-188.

5. N. Dunford, Spectral operators, Pacific J. Math. 4 (1954), 321-354.

6. C. FoIAş, Spectral maximal spaces and decomposable operators in Banach spaces, Archiv der Math. 14 (1963), 341-349.

7. C. Foraş, Une application des distributions vectorielles à la théorie spectrale, Bull. Sci. Math. 84 (1960), 147-158.

8. P. R. Harmos, Quasitriangular operators, Acta Sci. Math-Szeged 29 (1968), 283-294.

9. P. MEYER-NIEBERG, Quasitriangularerbare Operatoren und invarianten Untervektorräume stetiger linearer Operatoren, Archiv de Math. 22 (1971), 186-199.

10. C. Pearcy \& N. Salinas, An invariant subspace theorem, (pre-print).

11. B. Sz-Nagy \& C. Foraş, Sur les contractions de l'espace de Hilbert. IX. Factorisation de la fonction caracteristique. Sous-espaces invariants, Acta Sci. Math Szeged 25 (1964), 283-316.

12. B. Sz-Nagy \& C. Foraş, Analyse Harmonique des Opérateurs de l'Espace de Hilbert, Budapest, 1967.

13. P. R. Haxmos, Capacity in Banach algebra, Indiana Univ. Math. J. 20 (1971), 855-863.

Academie de la Republique Socialiste de Roumanie

Date communicated: JULY 5, 1972 